\title{
Dual-comb laser system for time-resolved studies of fireballs in the MIR
}

\author{
Ryan T. Rhoades ${ }^{1}$, Caroline Lecaplain ${ }^{1}$, Peter G. Schunemann², R. Jason Jones ${ }^{1}$ \\ 1. College of Optical Sciences, University of Arizona, 1630 E. University Blvd, Tucson AZ 85721 USA \\ 2. BAE Systems, P.O. Box 868 Nashua, New Hampshire 03063, USA \\ Email: rjjones@optics.arizona.edu
}

\begin{abstract}
We present a high power all-fiber dual-comb system suitable for time-resolved spectroscopy measurements in the mid-infrared to study explosion dynamics and detection of chemical species.

OCIS codes: (300.6340) Spectroscopy, infrared; (300.6500) Spectroscopy, time-resolved; (140.3510) Lasers, fiber
\end{abstract}

The development of spectroscopic techniques in the mid-infrared (MIR) is a very important area of investigation due to the wide variety of immediate applications of chemical detection in this wavelength region. Known as the molecular fingerprint region where there are strong molecular absorption cross-sections, absorption spectroscopy at this wavelength is extremely desirable for atmospheric monitoring, trace-chemical detection, and particularly characterization of fireballs and explosions. Due to the reduced optical opacity, fireball interiors may be penetrable in the MIR using high power coherent laser sources for absorption-based spectroscopy, which has largely been underexplored due to a lack of suitable sources [1]. Broadband absorption spectroscopy in the MIR with high power laser sources has the potential to probe inside fireballs and identify key molecular signatures even in the presence of continuum emission, which allows one to investigate parameters like the concentrations of added chemical species, combustion dynamics, and temperature fields within the fireball's interior.

To measure highly transient events in a fireball, we propose a new application of time-resolved dual-comb spectroscopy (DCS). The technique of dual-comb spectroscopy (DCS) is a robust implementation of performing spectrally and temporally resolved absorption spectroscopy. The strength of this method comes from the ability to generate optical delays through the interference of two frequency combs with slightly different repetition rates [2]. This inherently allows for the immediate conversion of optical spectroscopic information to be encoded into the RF which can be recovered using conventional detectors and electronics. When combined with the capacity for highpower scalability, which can be achieved with a fiber-optic platform, DCS offers many distinct advantages for performing highly time-resolved experiments in the MIR.

In order to develop a high power coherent dual comb system in the MIR, a method of generating MIR light through difference-frequency generation (DFG) of $1550 \mathrm{~nm}$ and $1950 \mathrm{~nm}$ generated from the same frequency comb source is employed [3]. First, two mode-locked Er-fiber oscillators were constructed using a nonlinear polarization evolution mode-locking mechanism, each with slightly tunable repetition rates near $74.7 \mathrm{MHz}$ and average output powers of $3 \mathrm{~mW}$. Both oscillators are pumped using the same $978 \mathrm{~nm}$ pump diode at high current, such that both are experiencing the same common noise. Additionally, the oscillators contain electro-optic modulation crystals and PZT-equipped

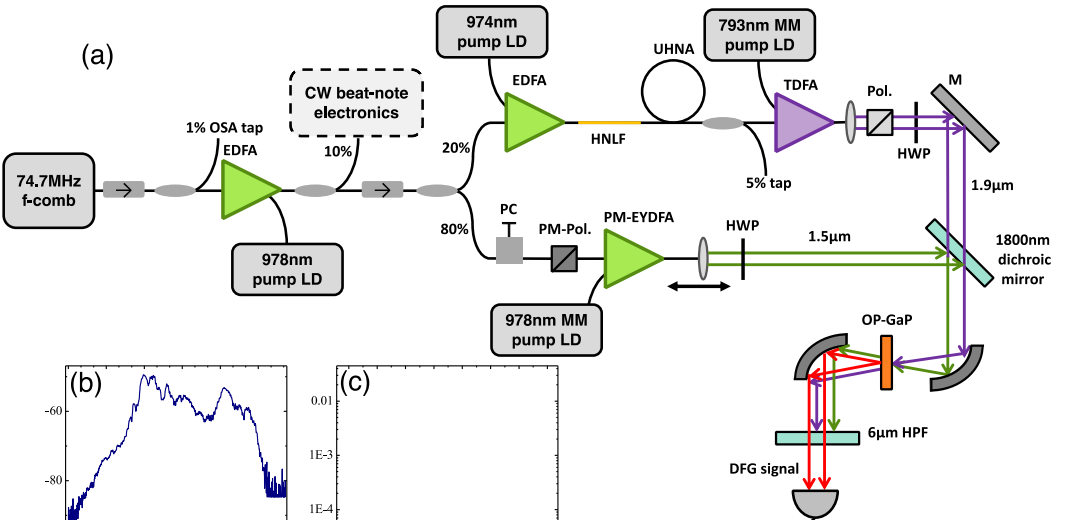

Figure 1. (a) Schematic diagram of MIR-generation setup starting from a mode-locked frequency comb, through $1.5 \mu \mathrm{m}$ and $1.95 \mu \mathrm{m}$ amplification arms, until difference frequency generation. EDFA, Er-doped fiber amplifier; PC, polarization controller; PM, polarization maintaining; Pol., polarizer; HNLF, highly nonlinear fiber; UHNA, ultrahigh NA fiber; TDFA, Tm-doped fiber amplifier; EYDFA, Er-Yb-doped fiber amplifier; HWP, half-wave plate; OP-GaP, orientation-patterned gallium phosphide; HPF, high-pass filter; DFG, difference frequency generation; MM, multi-mode; LD, laser

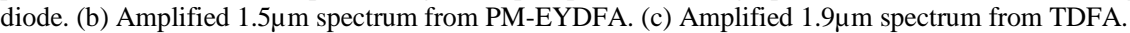


translation stages in a free-space section of the fiber laser to enable electronic phase-stabilization of the two oscillators' repetition rates. The outputs of these lasers are then passed through a Erbium-doped fiber amplifier (EDFA), increasing the output power to $103 \mathrm{~mW}$. The individual frequency combs are then split (see figure 1) and passed through a splitter to take $10 \%$ of the comb to mix and filter with a narrow line-width CW laser at $1561.424 \mathrm{~nm}$ to produce an optical beat-note used to phase-lock the oscillators, with the rest used in generating the MIR frequency-comb. Of this output, $20 \%$ is split and passed through a second anomalously dispersive $75 \mathrm{~cm}$ long EDFA to increase the power to $140 \mathrm{~mW}$ as well as to assist in compressing the pulse to about $160 \mathrm{fs}$. These compressed pulses are passed through $13 \mathrm{~cm}$ of $\mathrm{D}=1.2 \mathrm{ps} / \mathrm{nm} / \mathrm{km}$ highly nonlinear fiber to generate a coherent supercontinuum extending the frequency-comb bandwidth to $2.1 \mu \mathrm{m}$, and then $170 \mathrm{~cm}$ of normally dispersive ultrahigh NA fiber to pre-chirp the pulse train to seed a $106 \mathrm{~cm}$ long double-clad thulium-doped fiber amplifier. This results in a $630 \mathrm{~mW}, 140 \mathrm{fs}$ pulse centered at near $1900 \mathrm{~nm}$. The $80 \%$ portion of the split light is passed instead through a polarization controller and in-line polarizer, to optimize the output power to $42 \mathrm{~mW}$ of linearly polarized light, to seed a $2.1 \mathrm{~m}$ long polarization maintaining double-clad erbium-ytterbium fiber amplifier, resulting in $875 \mathrm{~mW}$ of $1560 \mathrm{~nm}$ light. The free-space outputs of these fiber-based amplification schemes are passed through polarization optics to optimize MIR generation, then combined on a dichroic mirror to spatially overlap the laser beams.

To generate the desired frequency comb in the MIR, various DFG crystals were tested, and the best frequency conversion results were achieved through using a $2.1 \mathrm{~mm}$ thick orientation-patterned gallium phosphide (OP-GaP) crystal. Using two parabolic gold mirrors, the two co-linear comb frequencies are focused onto the crystal and the generated DFG signal is subsequently collected and collimated. The DFG light is then imaged into a scanning monochromator. Figure 2 shows the spectrum centered near $8.3 \mu \mathrm{m}$ with a spectral width of $400 \mathrm{~nm}$.

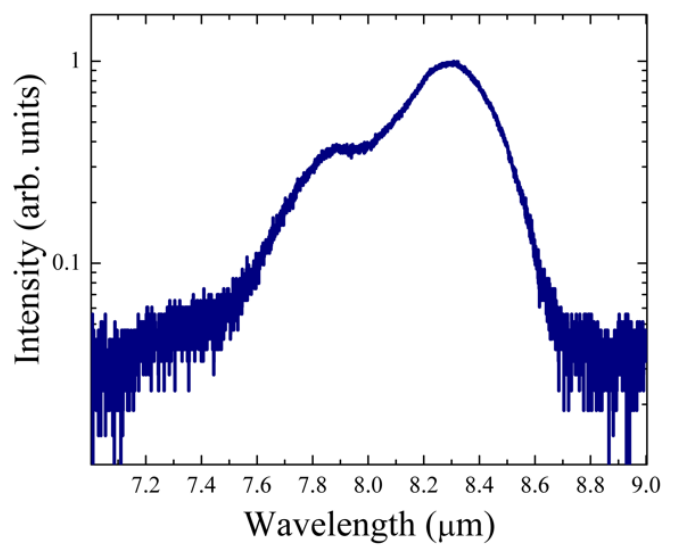

Figure 2. Generated DFG frequency comb spectrum from the erbium and thulium amplification scheme using an OP-GaP crystal.

The presented setup enables power scaling, due to having fiber-integrated amplification, as well as having the necessary control over the relative time-delay and phase-coherence of the two combs to achieve the desired timeresolved experimentation. These aspects of this particular DCS system are essential for creating a robust and powerful platform with the potential to study the highly transient characteristics present in combustion-based plasmas and, when appropriately scaled, explosive fireballs. This system will be used to demonstrate DCS in the MIR for time-resolved studies of species within fireball environments. Molecular signatures of chemicals like H2O, CO, CO2, NOx, and AlO can be detected to determine the time-evolving temperature and composition within explosions (or in laser generated plasma surrogates), as well as chemical weapon surrogates such as DMMP and DIMP.

Acknowledgements This material is based upon work supported by the Defense Threat Reduction Agency under Grant No. HDTRA 11710030.

\section{References}

[1] Nick Glumac "Absorption spectroscopy measurements in optically dense explosive fireballs using a modeless broadband dye laser." Applied spectroscopy 63, no. 9 (2009): 1075-1080.

[2] Ian Coddington, Nathan Newbury, and William Swann. "Dual-comb spectroscopy." Optica 3, no. 4 (2016): 414-426.

[3] Kevin F. Lee, Christopher J. Hensley, Peter G. Schunemann, and M. E. Fermann. "Midinfrared frequency comb by difference frequency of erbium and thulium fiber lasers in orientation-patterned gallium phosphide." Optics express 25, no. 15 (2017): 17411-17416. 